\title{
¿DESPEGA EL M-LEARNING? ANÁLISIS DE LA DISPOSICIÓN Y HÁBITOS DE LOS USUARIOS
}

\section{DOES THE M-LEARNING TAKE OFF? ANALYSIS OF USERS' DISPOSITION AND HABITS}

\author{
Dra. Carmen Rocío Yot Domínguez \\ carmenyot@us.es \\ Dr. Carlos Marcelo García \\ marcelo@us.es

\begin{abstract}
Universidad de Sevilla. Facultad de Ciencias de la Educación.
Departamento Didáctica y Organización Educativa. Pirotecnia s/n41018 Sevilla (España)
\end{abstract}

El m-learning se ha ido implantado en nuestro lenguaje como una realidad. Sin embargo, existen pocos estudios que analicen la aceptación por parte de los usuarios y de la disposición que tienen para formarse en este medio y en movilidad. El estudio que presentamos tiene por objetivo describir los hábitos de uso de la tecnología móvil de las personas en diferentes situaciones y contextos para confirmar la posible predisposición de los sujetos hacia el aprendizaje móvil. Para dar respuesta a nuestro objetivo diseñamos un instrumento de recogida de datos compuesto por las siguientes variables: sexo, edad y país; dispositivos electrónicos de que dispone; nivel de uso de cada uno de los dispositivos electrónicos en tres momentos diferenciados: en el trabajo, en formación y en tiempo de ocio; lugares en que utiliza cada uno de los dispositivos y tipo de uso que hace de cada uno de los dispositivos. Los resultados hallados nos muestran la baja penetración que la tecnología móvil tiene en los procesos formativos en la actualidad al tiempo que nos descubre dos cuestiones relevantes para el diseño y desarrollo de esta opción de formación, a saber: la necesidad de que ésta se adapte al contexto y de que las actividades de aprendizaje se ajusten a los hábitos de las personas al usar diferentes dispositivos.

Palabras claves: Aprendizaje móvil, dispositivo, tecnología, movilidad.

M-learning has been implanted in our language as a reality. However, there are few studies that analyse the acceptance by users of mobile devices in this training mode, supported by the use of mobile for its implementation, and layout to form in this environment and mobility. This study we present aims to describe the habits of use of mobile technology for people in different situations and contexts to confirm possible predisposition toward mobile learning subjects. To respond to our goal we design a data collection instrument made up of the following variables: sex, age and country; Electronic devices available; Level of use of electronic devices at three different times: in work, training and leisure time; Locations where each of the devices and use that makes each one of the devices. The results found show the low penetration that mobile technology currently has in the educational process at the time that reveals two issues relevant to the design and development of this training option, namely: the need that this fits the context and of learning activities conform to the known habits of use of devices.

Keywords: Mobile learning, device, technology, mobility.

Píxel-Bit. Revista de Medios y Educación. No 46. Enero 2015. ISSN: 1133-8482. e-ISSN: 2171-7966. doi: http://dx.doi.org/10.12795/pixelbit.2015.i46.13 


\section{Introducción.}

El auge que en los últimos años ha tenido el uso de los dispositivos móviles, su integración plena en la vida de las personas, junto a los continuos, y acertados, avances experimentados en su hardware, así como el desarrollo expansivo de las redes inalámbricas, han sido claves para la aparición del «mobile learning» (Caudill, 2007; Sharples \& Beale, 2003; Wagner, 2005).

Por «mobile learning» (m-learning, en adelante) se entiende aquel aprendizaje que se desarrolla total o parcialmente utilizando dispositivos móviles (Dyson, Litchfield, Lawrence, Raban \& Leijdekkers, 2009).El mlearning supone, según Georgiev, Georgieva y Smrikarov (2004), la capacidad de aprender en todas partes y en todo momento, sin conexión física permanente a las redes, mediante el uso de dispositivos móviles y portátiles como los teléfonos móviles, smart phones, ordenadores portátiles o tabletas. A este respecto, cabe matizar que no existe pleno consenso sobre la concepción del ordenador portátil como dispositivo móvil. Si bien son capaces de trabajar sin conexión a una fuente de energía y pueden utilizar las redes inalámbricas, no son dispositivos que las personas puedan llevar a todas partes y al que puedan acceder rápidamente en cualquier momento debido a su tamaño, su configuración y el tiempo requerido para arrancar y apagarse. En consecuencia, no se ajustan a lo que Livingston (2004) denomina «Dispositivo Móvil de Acceso a Internet» para aludir a aquellos dispositivos capaces de conectarse a Internet y comunicarse con otros dispositivos a través de la red, especialmente a los servidores, usando los protocolos estándar y que son lo suficientemente pequeños para caber cómodamente en un bol- sillo y convenientemente mantenerse con nosotros en todo momento.

El m-learning, pues, se diferencia del elearning en la medida en que se facilita a través de las tecnologías móviles para estudiantes que son también móviles (Koszalka \& Ntloedibe Kuswani, 2010). Y esta tecnología móvil se puede describir como todo dispositivo equipado con capacidad de comunicación inalámbrica pero a la vez pequeño, ligero, fácil de llevar y que nos acompaña en cada momento de nuestra vida cotidiana (Caudill, 2007; Chang, Sheu \& Chan, 2003; Fombona, Pascual \& Madeira, 2012).

Esta conceptualización del m-learning, adoleciendo de ser una definición tecnocéntrica (Winters, 2006), destaca más los aspectos tecnológicos de esta nueva modalidad formativa que la propia experiencia de aprendizaje subyacente la cual, según Traxler (2009), quedaría caracterizada como personal, informal, situada y sensible al contexto. Se corresponde esta aproximación con la primera de las trescategorías que han identificado Crescente y Lee (2011) para organizar todas las aportaciones que en la literatura han tratado de definir el m-learning y, verdaderamente, éstapoco ayuda a comprenderla naturaleza del aprendizaje móvil (Kukulska, Sharples, Milrad, Arnedillo \& Vavoula, 2011). Sin embargo, nos hace tomar conciencia delas nuevas características del medio, las cuales condicionan el diseño instruccional y los recursos de aprendizaje. Además, nos alerta sobre la necesidad de abordar el estudio de los factores que provocan la aceptación de los sistemas de aprendizaje móvil, por parte de los alumnos como cuestión clave para indirectamente potenciar los resultados de aprendizaje (Chen \& Huang, 2010; Liaw, Hatala \& Huang, 2010). 
La movilidad que proporciona m-learning, no sólo se refiere a los movimientos físicos o geográficos (Kakihara \& Sørensen, 2002), sino que tal como plantean Sharples, Arnedillo, Milrad, \& Vavoula (2007) puede incluir:

- Movilidad en el espacio físico. Las personas en continuo movimiento tratan de llevar el aprendizaje a un hueco de la vida diaria o utilizar sus momentos «libres» para reflexionar sobre lo que la vida diaria les ha enseñado. La ubicación puede ser así relevante para el aprendizaje o simplemente un telón de fondo.

- Movilidad de la tecnología. Las herramientas y los recursos están disponibles para ser llevados a todas partes convenientemente empaquetados en un único dispositivo ligero. A esto se une que en la actualidad es posible alternar entre distintos dispositivos.

- Movilidad en el espacio conceptual. Las personas pueden llegar a vivir numerosos episodios de aprendizaje todos los días, por lo que se mueve la atención de un tema conceptual a otro impulsado por el interés personal, la curiosidad o el compromiso.

- Movilidad en el espacio social. Las personas se mueven dentro de distintos grupos sociales, como la familia y/o los compañeros de oficina o aula.

- Dispersión en el tiempo del aprendizaje. El aprendizaje es un proceso acumulativo que mantiene vínculos entre, y se fortalece de, una variedad de experiencias de aprendizaje a través de contextos de aprendizaje formal e informal.

Teniendo esto presente, como apunta Kukulska Hulme (2010), el aprendizaje móvil nos desafía a tener que entender las circunstancias, necesidades y capacidades del alumnado, previo a la puesta en marcha de una acción formativa «mobile», lo que se ex- tiende a: 1) comprender cómo el aprendizaje ocurre en el curso de las rutinas diarias, entre desplazamientos y viajes, en la intersección de la vida personal, el trabajo, la formación y el ocio y 2) comprender las experiencias previas de las personas con las tecnologías móviles ya sea en el hogar, en el trabajo o para el entretenimiento, dado que pueden modelar las expectativas de éstas sobre lo que puede hacerse con un dispositivo móvil o de lo que constituye el aprendizaje móvil.

$\mathrm{Y}$ aquí encuentran su justificación trabajos como el de Clough, Jones, McAndrew \& Scanlon (2008) quienes estudiaron cómo los usuarios de los dispositivos móviles los utilizan y qué acciones de las que realizan favorecen, y cómo, el aprendizaje informal. También Hashim, Tan y Rashid (2014) que se propusieron analizar qué motiva a los adultos a participar en formación m-learning abordando la correlación existente entre la intención y las actividades usuales que realizan con sus dispositivos y que están destinadas a cubrir necesidades afectivas, cognitivas y de interacción social.

Disponer de esta información junto a la relativa a las preferencias de uso de los dispositivos móviles (para qué utilizan cada uno de ellos, en qué situaciones, en compañía de quién, etc.) es de utilidad asimismo a la hora de diseñary desarrollar sistemas para el aprendizaje móvil que sean personalizados y sensibles al contexto (Sampson \& Zervas, 2013; Zervas, Gómez, Fabregat \& Sampson, 2011).

\section{Metodología.}

El estudio que presentamos tiene por objetivo describir los hábitos de uso de la tecnología móvil de las personas en diferentes situaciones y contextos para confirmar la posible predisposición de los sujetos hacia el 
aprendizaje móvil y disponer de información contextual que sea de utilidad en el diseño e implementación de sistemas de formación móvil.

Para dar respuesta a nuestro objetivo diseñamos un instrumento de recogida de datos compuesto por las siguientes variables:

1) Sexo, edad y país.

2) Dispositivos electrónicos de que dispone.

3) Nivel de uso de cada uno de los dispositivos electrónicos en tres momentos diferenciados: en el trabajo, en formación y en tiempo de ocio.

4) Lugares en que utiliza cada uno de los dispositivos cuando está trabajando, cuando realiza algún tipo de formación o cuando dispone de tiempo libre.

5) Tipo de uso que hace de cada uno de los dispositivos cuando se está trabajando, cuando se realiza algún tipo de formación o cuando se encuentra de ocio: hacer fotos, enviar/recibir fotos, grabar vídeos, enviar/recibir vídeos, grabar audios, enviar/recibir audios, escuchar música, enviar correo electrónico, recibir correo electrónico, chatear, enviar/recibir mensajes instantáneos, escribir notas, etc.

6) Con quién se está cuando se hace uso de los diferentes dispositivos: en el trabajo, en la formación o cuando se dispone de tiempo libre.

7) Disposición hacia el aprendizaje móvil.

Tras su redacción, el instrumento fue sometido a un juicio de expertos para valorar su estructura interna y el enunciado de cada una de las preguntas. Era necesario, previo a proceder a la recogida de datos, garantizar que quedaban diferenciados los interrogantes en función de los momentos seleccionados y no se generaba confusión al tratar de responderlo.

Una vez que dispusimos de su versión final, se diseñó el instrumento en la aplicación online e-encuestas, se distribuyó a través de la web y permaneció abierto para ser respondido durante los meses de abril y mayo del año 2014. Al tratarse de un instrumento de recogida de datos electrónico, utilizamos diferentes medios para asegurarnos de su adecuada difusión. Se ha informado del estudio así como solicitado participación y colaboración a través de una lista de distribución (Telefpo) y diferentes redes sociales y profesionales (Red Linkedin o Facebook).

Los datos recogidos fueron analizados estadísticamente haciendo uso del software SPSS. Sobre ellos se aplicó un análisis descriptivo en base al recuento de frecuencias y porcentaje de respuesta a cada una de las preguntas, así como un análisis bivariable que buscaba la relación entre las distintas variables consideradas.

Al inventario, para medir su fiabilidad, se le calculó el coeficiente Alfa de Cronbach. El coeficiente de consistencia interna obtenido ha sido de .962 . Si lo aplicamos de manera diferenciada a las preguntas relacionadas a cada uno de los momentos que hemos identificado (cuando se está trabajando, cuando se está formando o cuando se encuentra disfrutando del tiempo libre) el coeficiente se mantiene en valores altos, a saber: .936; .971 y .969; respectivamente.

En total, 384 personas lo han cumplimentado. De ellos, 193 (50.3\%) son hombres y 191 (49.7\%) son mujeres. Sus edades oscilan entre los 21 años y más de 60 años, siendo los tres tramos de edad más frecuentes entre la muestra los que siguen: 26-30 (15.6\%), 31-35 (18.0\%) y $36-40(20.6 \%)$. El $70.8 \%$ de la muestra reside en España, del resto la mayoría lo 


\begin{tabular}{|c|c|c|c|c|c|c|c|}
\hline & & FRECUENCIA & PORCENTAJE & & & FRECUENCIA & PORCENTAJE \\
\hline \multirow[t]{4}{*}{ País } & Argentina & 18 & 4.7 & \multirow[t]{4}{*}{ Edad: } & $26-30$ & 60 & 15.6 \\
\hline & Chile & 39 & 10.2 & & $31-35$ & 69 & 18.0 \\
\hline & Colombia & 14 & 3.6 & & $36-40$ & 79 & 20.6 \\
\hline & España & 272 & 70.8 & & $41-45$ & 56 & 14.6 \\
\hline
\end{tabular}

Tabla 1. Tabla de frecuencia de las variables país y edad.

hace en América Latina así el 10.2\% se localiza en Chile, el $4.7 \%$ en Argentina y el $3.6 \%$ en Colombia. Véase Tabla 1.

Más del $70 \%$ de las personas encuestadas dispone de televisión (93.8\%), ordenador portátil $(95.6 \%)$, ordenador de sobremesa $(74.5 \%)$ o teléfono móvil $(79.4 \%)$. En torno al $65 \%$ tiene un smart phone $(68.5 \%)$ o reproductor de podcasts $(65.1 \%)$ y menos del $40 \%$ tablets $(30.2 \%)$ o videoconsolas $(39.1 \%)$. Entre otros dispositivos electrónicos que los sujetos afirman disponer se citan: las cámaras fotográficas y de video, el Sistema de Posicionamiento Global (o GPS) o los e-reader (o lector de libro electrónico).

\section{Resultados.}

\subsection{Uso que se hace de los dispositivos en el puesto de trabajo.}

En relación con el uso que las personas hacen de los dispositivos móviles en el puesto de trabajo, observamos que mientras que el $80.2 \%$ de la muestra confirma que hace uso habitualmente del ordenador portátil cuando trabaja, sólo el 55.5\% utiliza el smart phone o el $50.6 \%$ el teléfono móvil. Como se observa en la Tabla 2, más de la mitad de las personas que tienen ordenador portátil en su puesto de trabajo con asiduidad también cuentan con el teléfono móvil y/o el smart phone.

Por otra parte, menos del $20 \%$ de los encuestados usa la tablet $(15.6 \%)$, el reproductor de podcast $(18 \%)$ o la videoconsola (3.2\%) trabajando. La práctica totalidad de los sujetos que disponen con normalidad de la tablet cuenta también con un ordenador portátil y la mayoría con teléfono móvil. Véase Tabla 3.

Podemos indicar que, según las salidas de la prueba chi-cuadrado, el nivel de uso que se hace de todos estos dispositivos ha resultado no ser dependiente del sexo, la edad o el país de procedencia de los encuestados.

El ordenador portátil se utiliza con mayor frecuencia en el hogar (78.6\%) que en la oficina $(55.5 \%)$ cuando se trabaja. Por su parte, el ordenador de sobremesa se emplea más en la oficina (67.2\%). El smart phone es utilizado prácticamente de manera similar en la oficina,

\begin{tabular}{|lr|r|r|r|r|r|r|}
\hline & \multicolumn{3}{|c|}{ TELÉFONO MÓVIL } & \multicolumn{3}{c|}{ SMART PHONE } \\
\cline { 3 - 8 } & & Frecuente & $\begin{array}{c}\text { Muy } \\
\text { frecuente }\end{array}$ & Del total & Frecuente & $\begin{array}{c}\text { Muy } \\
\text { frecuente }\end{array}$ & Del total \\
\hline Ordenador & Frecuente & $13(23.2 \%)$ & $11(19.6 \%)$ & $56(100 \%)$ & $12(21.4 \%)$ & $17(30.4 \%)$ & $56(100 \%)$ \\
portátil & Muy frecuente & $16(6.3 \%)$ & $122(48.4 \%)$ & $252(100 \%)$ & $28(11.1 \%)$ & $125(49.6 \%)$ & $252(100 \%)$ \\
Del total & & 44 & 150 & & 54 & 159 & \\
\hline
\end{tabular}

Tabla 2. Tabla de correlación de la variable uso del ordenador portátil y las variables uso del teléfono móvil y uso del smart phone mientras se encuentra el usuario trabajando. 


\begin{tabular}{|lr|r|r|r|r|r|r|}
\hline & \multicolumn{3}{|c|}{ ORDENADOR PORTÁTIL } & \multicolumn{3}{c|}{ TELÉFONO MÓVIL } \\
& & Frecuente & $\begin{array}{c}\text { Muy } \\
\text { frecuente }\end{array}$ & Del total & Frecuente & \multicolumn{1}{c|}{$\begin{array}{c}\text { Muy } \\
\text { frecuente }\end{array}$} & Del total \\
\hline Tablet & Frecuente & $4(17.4 \%)$ & $18(78.3 \%)$ & $23(100 \%)$ & $4(17.4 \%)$ & $10(43.5 \%)$ & $23(100 \%)$ \\
& Muy frecuente & $2(5.4 \%)$ & $32(86.5 \%)$ & $37(100 \%)$ & $2(5.4 \%)$ & $24(64.9 \%)$ & $37(100 \%)$ \\
Del total & & 56 & 252 & & 44 & 150 & \\
\hline
\end{tabular}

Tabla 3. Tabla de correlación de la variable uso de la tablet y las variables uso del teléfono móvil y uso del ordenador portátil mientras se encuentra el usuario trabajando.

\begin{tabular}{|r|r|c|r|r|r|r|r|r|r|r|}
\hline & \multicolumn{2}{|c|}{} & \multicolumn{2}{c|}{ EN } & \multicolumn{2}{c|}{ EN } & \multicolumn{2}{c|}{} \\
& \multicolumn{2}{|c|}{ EN LA OFICINA } & \multicolumn{2}{c|}{ EN CASA } & \multicolumn{2}{c|}{ TRANSPORTE } & \multicolumn{2}{c|}{ CAFETERÍAS } & \multicolumn{2}{c|}{ EN CALLE } \\
\cline { 2 - 10 } & Frecuencia & $\%$ & Frecuencia & $\%$ & Frecuencia & $\%$ & Frecuencia & $\%$ & Frecuencia & $\%$ \\
\hline Ordenador & 258 & 67.2 & 166 & 43.2 & - & - & - & - & - & - \\
Portátil & 213 & 55.5 & 302 & 78.6 & 40 & 10.4 & 100 & 26.0 & 14 & 3.6 \\
Smart phone & 199 & 51.8 & 221 & 57.6 & 220 & 57.3 & 235 & 61.2 & 249 & 64.8 \\
Tablet & 39 & 10.2 & 94 & 24.5 & 34 & 8.9 & 52 & 13.5 & 20 & 5.2 \\
Reproductor & 40 & 10.4 & 115 & 29.9 & 120 & 31.3 & 55 & 14.3 & 110 & 28.6 \\
\hline
\end{tabular}

Tabla 4. Tabla de frecuencia de las variables uso del ordenador de sobremesa, ordenador portátil, smart phone, tablet y reproductor de podcast en la oficina, en casa, en el transporte público, en cafeterías y en la calle mientras se encuentra el usuario trabajando.

en casa, en el transporte público, en cafeterías o en la calle. Sí es cierto que es algo más frecuente su uso en estos dos últimos espacios que en los anteriores citados. Además, como se muestra en la Tabla 4, el smart phone es el dispositivo de uso predominante fuera de la oficina o del hogar.

Encontramos además que más del $60 \%$ de la muestra, en sus puestos de trabajo, envía emails $(65.9 \%)$ y consulta los recibidos
$(61.7 \%)$, trabaja con documentos $(68.5 \%)$ o bases de datos $(62.0 \%)$ y realiza búsquedas de información en internet $(66.7 \%)$ a través del ordenador de sobremesa. Sin embargo, el ordenador portátil es empleado por mayor número de encuestados para realizar las mismas acciones.

En lo que respecta al smart phone, como se observa en la Tabla 5, éste es utilizado por más del $50 \%$ de las personas para: hacer fo-

\begin{tabular}{|r|r|r|r|r|r|r|r|r|}
\hline & \multicolumn{2}{|c|}{ ORDENADOR } & \multicolumn{2}{|c|}{ PORTÁTIL } & \multicolumn{2}{c|}{ SMART PHONE } & \multicolumn{2}{|c|}{ TABLET } \\
\cline { 2 - 8 } & Frecuencia & $\%$ & Frecuencia & $\%$ & Frecuencia & $\%$ & Frecuencia & $\%$ \\
\hline Enviar emails & 253 & 65.9 & 299 & 77.9 & 199 & 51.8 & 68 & 17.7 \\
Recibir emails & 237 & 61.7 & 289 & 75.3 & 202 & 52.6 & 52.6 & 17.2 \\
Trabajar con documentos & 263 & 68.5 & 297 & 77.3 & 44 & 11.5 & 37 & 9.6 \\
Trabajar con base de datos & 238 & 62.0 & 258 & 67.2 & 13 & 3.4 & 12 & 3.1 \\
Realizar búsquedas en Internet & 256 & 66.7 & 302 & 78.6 & 173 & 45.1 & 72 & 18.8 \\
Hacer fotografías & 21 & 5.5 & 51 & 13.3 & 223 & 58.1 & 34 & 8.9 \\
Enviar mensajes instantáneos & 110 & 28.6 & 166 & 43.2 & 225 & 58.6 & 40 & 10.4 \\
Activar recordatorios & 108 & 28.1 & 128 & 33.3 & 201 & 52.3 & 35 & 9.1 \\
Gestionar la agenda & 147 & 38.3 & 199 & 51.8 & 197 & 51.3 & 50 & 13.0 \\
Acceder a redes sociales & 194 & 50.5 & 262 & 68.2 & 198 & 51.6 & 69 & 18.0 \\
\hline
\end{tabular}

Tabla 5. Tabla de frecuencia de las variables uso del ordenador de sobremesa, uso del ordenador portátil, uso del smart phone y uso de la tablet para diferentes acciones mientras se encuentra el usuario trabajando.

Píxel-Bit. Revista de Medios y Educación. No 46. Enero 2015. ISSN: 1133-8482. e-ISSN: 2171-7966. doi: http://dx.doi.org/10.12795/pixelbit.2015.i46.13 
tografías $(58.1 \%)$, enviar $(51.8 \%)$ y recibir $(52.6 \%)$ emails, enviar mensajes instantáneos $(58.6 \%)$, activar recordatorios $(52.3 \%)$ y gestionar la agenda $(51.3 \%)$ o acceder a las redes sociales $(51.6 \%)$. Con este dispositivo, sólo el $11.5 \%$ de los encuestados trabaja con documentos, el $3.4 \%$ con base de datos y el $6.8 \%$ con presentaciones. La tablet esencialmente se utiliza para leer, acceder a las redes sociales, realizar búsquedas en internet y revisar el email.

En consecuencia, para el envío y la recepción de emails se hace uso de los cuatros dispositivos. Sin embargo, más del 50\% de la muestra opta por el portátil para comunicarse sincrónicamente, por ejemplo para chatear $(52.6 \%)$ o hacer videoconferencias $(58.3 \%)$. E1 $71.4 \%$ de las personas afirman que cuando envían o consultan los emails recibidos, lo hacen sin compañía. Por otra parte, prácticamente el mismo número de encuestados confirma chatear solos (40.6\%) o acompañados de sus colegas $(38.3 \%)$.

Para trabajar con documentos y bases de datos esencialmente se opta por el ordenador de sobremesa o el portátil y éstas son actividades que el $81.0 \%$ y el $74.0 \%$ de la muestra, respectivamente, realiza de manera individual. Para tomar instantáneas se utiliza principalmente el smart phone y esto el mayor porcentaje de encuestados $(44.5 \%)$ señala hacerlo también sin compañía.

\subsection{Uso que se hace de los dispositivos durante la formación.}

En relación con el uso de los dispositivos móviles durante la formación, observamos que si bien el $62.2 \%$ de la muestra hace uso del ordenador portátil con regularidad cuando se forma, sólo el 17.2\% confirma utilizar el smart phone, el $12.2 \%$ el teléfono móvil y el $11.7 \%$ la tablet.Como se observa en las Tablas 6 y 7 , el $35 \%$ de los que dicen utilizar el ordenador portátil con frecuencia y más del $40 \%$ de los que lo emplean con muy alta frecuencia, no usan o lo hacen en muy baja medida el smart phone. Del mismo modo, no se asocia el uso frecuente del ordenador portátil con el de la tablet. Por su parte, el ordenador de sobremesa es utilizado por el $40.9 \%$ de los sujetos.

Cabe reseñarse que la frecuencia de uso de los diferentes dispositivos manifestada por los encuestados, atendiendo a los resultados de la prueba chi-cuadrado (véase Tabla

\begin{tabular}{|c|c|c|c|c|c|c|c|c|c|}
\hline & \multicolumn{7}{|c|}{ SMART PHONE } & \multirow[b]{2}{*}{ Del total } \\
\hline & & & 0 & 1 & 2 & 3 & 4 & 5 & \\
\hline Ordenador & & 9 & $9(22.5 \%)$ & $3(7.5 \%)$ & $2(5.0 \%)$ & $8(20.0 \%)$ & $3(7.5 \%)$ & $6(15.0 \%)$ & $40(100 \%)$ \\
\hline portátil & 5 & 36 & $61(30.7 \%)$ & $6(3.0 \%)$ & $15(7.5 \%)$ & $33(16.6 \%)$ & $19(9.5 \%)$ & $29(14.6 \%)$ & $199(100 \%)$ \\
\hline Del total & & 149 & 86 & 12 & 22 & 49 & 28 & 38 & 384 \\
\hline
\end{tabular}

Tabla 6. Tabla de relación de la variable uso del ordenador portátil y la variable uso del smart phone mientras se encuentra el usuario en formación.

\begin{tabular}{|c|c|c|c|c|c|c|c|c|c|}
\hline & \multicolumn{7}{|c|}{ TABLET } & \multirow[b]{2}{*}{ Del total } \\
\hline & & & 0 & 1 & 2 & 3 & 4 & 5 & \\
\hline Ordenador & & 14 & $15(37.5 \%)$ & $1(2.5 \%)$ & $4(10.0 \%)$ & $1(2.5 \%)$ & $2(5.0 \%)$ & $3(7.5 \%)$ & $40(100 \%)$ \\
\hline portátil & 5 & 68 & $79(39.7 \%)$ & $4(2.0 \%)$ & $5(2.5 \%)$ & $10(5.0 \%)$ & $17(8.5 \%)$ & $16(8.0 \%)$ & $199(100 \%)$ \\
\hline Del total & & 195 & 113 & 6 & 10 & 15 & 23 & 22 & 384 \\
\hline
\end{tabular}

Tabla 7. Tabla de relación de la variable uso del ordenador portátil y la variable uso de la Tablet mientras se encuentra el usuario en formación.

Píxel-Bit. Revista de Medios y Educación. No 46. Enero 2015. ISSN: 1133-8482. e-ISSN: 2171-7966. doi: http://dx.doi.org/10.12795/pixelbit.2015.i46.13 


\begin{tabular}{|llll|}
\hline & \multicolumn{3}{c|}{ Chi-cuadrado } \\
& País & Sexo & Edad \\
Ordenador de sobremesa & .801 & .474 & .578 \\
Ordenador portátil & .247 & .077 & .251 \\
Teléfono móvil & .407 & .384 & .289 \\
Smart phone & .657 & .647 & .800 \\
Tablet & .857 & .908 & .491 \\
\hline
\end{tabular}

Tabla 8. Prueba chi-cuadrado.

8), es independiente del sexo, la edad y el país de procedencia de los sujetos.

El ordenador de sobremesa es el dispositivo por el que opta mayor número de encuestados $(41.7 \%)$ cuando se forman y se encuentran en la oficina, mientras que el ordenador portátil pasa a ser el más empleado en el centro de formación (46.4\%) y en el hogar $(58.9 \%)$. En el transporte público $(25.3 \%)$, en cafeterías $(25.3 \%)$ y en la calle $(24.7 \%$ ) más del $24 \%$ de los sujetos afirma utilizar el smart phone con fines formativos, convirtiéndose además en el dispositivo más utilizado en estos espacios. La tablet donde es más utilizada es en el hogar. Véase Tabla 9.

En este caso, la prueba chi-cuadrado muestra dependencia entre el sexo de los encuestados y el uso del ordenador portátil en el centro de formación (sig. .018) y de la tablet en cafeterías (sig. .011). Así, el 59.7\% de las mujeres que respondieron el inventario informaron que no utilizaban el ordenador portátil en el centro de formación o el $94.8 \%$ de los hombres señalaron no usar la tablet en cafeterías.

Respecto al uso que hacen de estos dispositivos, más del $50 \%$ de las personas hace uso del ordenador portátil para enviar emails (52.9\%) o consultar los recibidos $(51.8 \%)$ y para trabajar con documentos $(52.6 \%)$. Para trabajar sobre bases de datos, crear o ver presentaciones o realizar búsquedas en internet más del $40 \%$ se decanta por emplear el ordenador portátil y más del $30 \%$ por el ordenador de sobremesa.

El smart phone es utilizado esencialmente para enviar y recibir mensajes instantáneos (24.5\%), recibir $(23.7 \%)$ y enviar emails (22.4\%), activar recordatorios o gestionar la agenda ( $21.4 \%$, respectivamente) y acceder a las redes sociales (20.1\%). Sólo el $4.2 \%$ de los encuestados trabaja con bases de datos desde él y el $5.2 \%$ y $6.2 \%$ con presentaciones y documentos respectivamente. La tablet se destina a la lectura. Véase tabla 10.

\begin{tabular}{|c|c|c|c|c|}
\hline & ORDENADOR & PORTÁTIL & SMART PHONE & TABLET \\
\hline & $\%$ & $\%$ & $\%$ & $\%$ \\
\hline$\overline{\text { Oficina }}$ & 41.7 & 37.2 & 17.7 & 7.6 \\
\hline Centro de formación & 23.2 & 46.4 & 17.7 & 8.1 \\
\hline Casa & 32.3 & 58.9 & 24.5 & 13.5 \\
\hline Transporte público & - & 7.6 & 25.3 & 6.5 \\
\hline Cafeterías & - & 14.1 & 25.3 & 8.9 \\
\hline Calle & & 2.3 & 24.7 & 2.9 \\
\hline
\end{tabular}

Tabla 9. Tabla de frecuencia de las variables uso del ordenador de sobremesa, ordenador portátil, smart phone y tablet en la oficina, en el centro de formación, en casa, en el transporte público, en cafeterías y en la calle mientras se encuentra el usuario en formación.

Píxel-Bit. Revista de Medios y Educación. No 46. Enero 2015. ISSN: 1133-8482. e-ISSN: 2171-7966. doi: http://dx.doi.org/10.12795/pixelbit.2015.i46.13 


\begin{tabular}{|r|r|r|r|r|}
\hline & ORDENADOR & PORTÁTIL & SMART PHONE & TABLET \\
\cline { 2 - 5 } & $\%$ & $\%$ & $\%$ & $\%$ \\
\hline Enviar emails & 33.3 & 52.9 & 22.4 & 8.9 \\
Recibir emails & 33.3 & 51.8 & 23.7 & 8.1 \\
Trabajar con documentos & 37.8 & 52.6 & 6.5 & 5.2 \\
Trabajar con base de datos & 31.3 & 42.7 & 4.2 & 2.9 \\
Realizar búsquedas en Internet & 33.6 & 49.7 & 19.5 & 8.3 \\
Ver/Crear presentaciones & 32.8 & 46.9 & 5.2 & 4.2 \\
Enviar mensajes instantáneos & 17.2 & 30.7 & 24.5 & 5.2 \\
Activar recordatorios & 15.1 & 23.4 & 21.4 & 4.9 \\
Gestionar la agenda & 16.4 & 28.1 & 21.4 & 6.8 \\
Acceder a redes sociales & 23.2 & 37.8 & 20.1 & 8.6 \\
\hline
\end{tabular}

Tabla 10. Tabla de frecuencia de las variables uso del ordenador de sobremesa, uso del ordenador portátil, uso del smart phone y uso de la tablet para diferentes acciones mientras se encuentra el usuario en formación.

Del mismo modo que en la situación anterior, el envío y recepción de emails se encuentra entre las tareas que se realiza con mayor frecuencia en cada uno de los cuatro dispositivos. Ambas acciones son realizadas sin compañía por más del $40 \%$ de los sujetos. También el trabajo con documentación, con bases de datos o presentaciones, la lectura o la búsqueda de información se realizan de manera individual.

\subsection{Uso que se hace de los dispositivos en el tiempo de ocio.}

A diferencia de las dos situaciones anteriores (formación o trabajo), en relación con el uso de dispositivos móviles en momentos de ocio, nos encontramos que el número de sujetos que utilizan el ordenador portátil (33.1\%) y el de sobremesa $(14.6 \%)$ desciende. En el tiempo de ocio, el smart phone
(31.8\%), pasa a ser de las tecnologías más empleadas, sólo superada en uso por el ordenador portátil. Les siguen la televisión (24.0\%) y el reproductor de podcasts $(16.9 \%)$.

De todos estos dispositivos, sólo el nivel de uso del PC (sig. .040) se muestra dependiente del sexo de los sujetos y la frecuencia de uso de la televisión (sig. .036) del país de residencia de los mismos. Sabemos, por ejemplo, que la mayoría de las personas que nunca utilizan la televisión en su tiempo de ocio son españolas. No obstante, el 50.4\% de los encuestados procedentes de España hacen uso regular o muy regular durante el tiempo de ocio de la televisión.

El smart phone es el dispositivo que más se utiliza en la calle, en cafeterías o en el transporte público. En el hogar el más usado es el portátil, seguido por el smart phone y la TV. Véase Tabla 11.

\begin{tabular}{|l|r|r|r|r|}
\hline & EN CASA & EN CALLE & EN CAFETERÍAS & EN TRANSPORTE \\
\cline { 2 - 5 } & \multicolumn{1}{|c|}{$\%$} & $\%$ & $\%$ & $\%$ \\
\hline Portátil & 44.5 & 2.9 & 9.1 & 4.2 \\
Smart phone & 33.3 & 28.1 & 29.4 & 26.8 \\
TV & 29.9 & - & 1.6 & - \\
Reproductor & 18.2 & 14.6 & 10.9 & 12.0 \\
\hline
\end{tabular}

Tabla 11. Tabla de frecuencia de las variables uso del ordenador portátil, smart phone, $T V$ y reproductor de podcast en casa, en el transporte público, en cafeterías y en la calle durante el tiempo de ocio 


\begin{tabular}{|r|r|r|r|}
\hline & Portátil & Smart phone & Tablet \\
\cline { 2 - 4 } & $\%$ & $\%$ & $\%$ \\
\hline Hacer fotografías & 12 & 31.8 & 5.5 \\
Enviar emails & 32.8 & 29.4 & 8.9 \\
Recibir emails & 32.8 & 28.6 & 8.9 \\
Realizar búsquedas en Internet & 28.9 & 22.1 & 6.5 \\
Enviar fotografías & 26.8 & 28.6 & 6.8 \\
Acceder a redes sociales & 29.9 & 24.2 & 8.6 \\
\hline
\end{tabular}

Tabla 12. Tabla de frecuencia de las variables uso del ordenador portátil, uso del smart phone y uso de la tablet para diferentes acciones durante el tiempo de ocio.

Las cuatro acciones más realizadas por los encuestados a través del smart phone en su tiempo libre son: hacer fotografías $(31.8 \%)$, enviar emails (29.4\%), recibir emails (28.6\%) y enviar fotografías (28.6\%). El envío y recepción de emails se encuentran, asimismo, entre las que mayor número de sujetos hacen desde el ordenador portátil, seguidas del acceso a redes sociales $(29.9 \%)$ y la búsqueda de información en internet (28.9\%).Véase Tabla 12 .

Si bien todas ellas se caracterizan porque los sujetos las desarrollan esencialmente en solitario, nos encontramos que la toma de instantáneas es ahora más frecuente que se desarrolle en compañía de la familia.

\section{Discusión.}

A la luz de los resultados expuestos, la primera conclusión que hemos de destacar es el bajo nivel de uso que se hace de la tecnología móvil en los procesos formativos en la actualidad.A pesar del liderazgo que los dispositivos móviles como tecnología dispone en el mundo y los cada vez mayores niveles de penetración de la banda ancha móvil, según los análisis y prospectivas de diferentes organismos como la Unión Internacional de Telecomunicaciones (UIT, 2011), nos encontramos que el smart phone, aun siendo de ellos el más utilizado con fines formativos, lo es por un escaso $17 \%$ del total de las perso- nas encuestadas. Y, cabe subrayar, que esta tendencia no es dependiente ni de la edad, ni del sexo, ni del lugar de procedencia de los usuarios.

Tsinakos y Ally (2014), después de analizar el estado del mobile learning en siete de las regiones del mundo confirman que la tasa de adopción del aprendizaje móvil no evoluciona al mismo ritmo frenético que ha alcanzado la irrupción de la tecnología móvil en la vida de los usuarios a nivel mundial. Resultados similares se encontraron a través del estudio desarrollado por el Observatorio de la Formación en Red SCOPEO (Camacho \& Lara, 2011) en España, Portugal y América Latina para dar a conocer las iniciativas que se están implementando en esta modalidad formativa.

Sin embargo, en el día a día la adopción de este tipo de dispositivo queda relegada más bien al contexto laboral donde más de la mitad de los encuestados ya los han integrado en sus quehaceres con regularidad o al de ocio donde se convierte en la tecnología de uso preferente. Ahora bien, sea en uno u otro momento, el patrón de uso dado a los dispositivos móviles es el mismo. Y ahí se encuentra la información de mayor interés para la finalidad última de este estudio.

En primer lugar, por contribuir a la toma de conciencia de que, en m-learning, la entrega del material de estudio, la presentación de las actividades de aprendizaje y el apoyo a los

Píxel-Bit. Revista de Medios y Educación. No 46. Enero 2015. ISSN: 1133-8482. e-ISSN: 2171-7966. doi: http://dx.doi.org/10.12795/pixelbit.2015.i46.13 
estudiantes deben ofrecerse adaptados no sólo al dispositivo en cuestión sino también atendiendo al contexto entendido al menos como ubicación (lugar donde se ubica la persona), disponibilidad temporal (tiempo del que dispone para el aprendizaje) y conducta (actividad/es que se encuentra realizando). Tal como ha sido expuesto, el smart phone es la tecnología más utilizada al aire libre mientras se pasea o nos trasladamos en transporte público, así como en cafeterías. En definitiva, en situaciones en las que las condiciones ambientales son diferentes y altamente cambiantes (como la luz, el sonido o en su defecto ruido, etc.) y en las que se compagina el uso de la tecnología con otras actividades de diferenciado grado de movilidad física por parte de los sujetosy requerimientos de atención (como correr, pasear, encontrase tomando un café, estar esperando la llegada del tren, etc.). La tablet donde más se utiliza es en el hogar y aquí comparte presencia principalmente con el ordenador portátil.

En segundo, por darnos a conocer las actividades de aprendizaje que serán más aceptadas en función del dispositivo en el que se distribuyan a sabiendas de que aún no estamos en condiciones de sustituir los ordenadores de sobremesa o portátiles. Hemos visto que se opta por la tablet para la lectura o la realización de búsquedas en internet mientras que el smart phone, puede que motivado por su particularidad de teléfono, es destinado a actividades comunicativas como enviar y recibir un email o mensajes instantáneos o acceder a una red social, también es empleado para tomar y enviar fotografías y para organizar nuestro tiempo activando recordatorios y gestionando nuestra agenda. A pesar de su capacidad para reproducir recursos audiovisuales o para permitir el trabajo con documentación, no son usados para este fin. Para esto aún se utiliza el ordenador ya sea de sobremesa o portátil.

En suma, los resultados hallados nos permiten entender mejor los hábitos de uso de la tecnología móvil, lo que nos facilita la tarea de diseño de sistemas de formación que distribuyan los contenidos y apoyos al estudio y favorezcan el desarrollo de actividades de aprendizaje en diferentes dispositivos ajustándose no sólo a nuestra preferencia de uso de cada uno de estos dispositivos en función de distintos momentos, sino también a las condiciones ambientales de nuestra situación geográfica, nuestra disponibilidad de tiempo y al hecho de si estamos compatibilizando el aprendizaje con otras actividades que nos estén ocupando y de qué tipo son éstas.

Los hallazgos que presentamos van en la línea de los recientes estudios sobre «context aware mobile learning», que destacan la necesidad de incluir el contexto en el que la persona está para ofrecerle recursos de aprendizaje. En este sentido, Chamasemani y Affendey (2014) categorizan diferentes tipos de contexto: contexto ambiental (condiciones ambientales de la ubicación actual del sujeto, tales como: la temperatura, el nivel de ruido y de luz), contexto espacial (información respecto a la posición de la persona y dispositivo), contexto de actividad (actividad actual del usuario incluyendo actividades privadas y profesionales que puedan ser percibidas como: hablando, caminando o corriendo), contexto personal (características del usuario como: edad, sexo, aficiones e intereses del usuario) y contexto temporal (como la estación del año, la fecha y hora actual). En esta línea, Anagnostopoulos, Tsounis y Hadjiefthymiades (2007) describen con amplitud el conjunto de características relacio- 
nadas con el modelado del contexto de una aplicación sensible al contexto.

En e-learning disponemos de un claro precedente de este tipo de sistemas, estos son, los sistemas hipermedias adaptativos. Pero con la salvedad de queestos esencialmente atienden a diferentes datos acerca de los usuarios (Brusilovsky, 2012)como sus estilos de aprendizaje (Akbulut \& Cardak, 2012). En el aprendizaje móvil han de tomarse en cuenta nuevas características en el proceso de adaptación (Chorfi, Sevkli \& Bousbahi, 2012) y todas ellas las hemos venido enumerando.

\section{Referencias bibliográficas.}

Akbulut, Y. \& Cardak, C. S. (2012). Adaptive educational hypermedia accommodating learning styles: A content analysis of publications from 2000 to 2011. Computers \& Education, 58 (2), 835-842. doi: 10.1016/ j.compedu.2011.10.008

Anagnostopoulos, C. B., Tsounis, A.\& Hadjiefthymiades, S. (2007). Context Awareness in Mobile Computing Environments. Wireless Personal Communications: An International Journal, 42 (3), 445-464. doi: 10.1007/s11277-006-9187-6

Brusilovsky, P. (2012). Adaptive Hypermedia for Education and Training. In P. J. Durlach \& A. M. Lesgold (Eds.). Adaptive Technologies for Training and Education. (p.46-66). Cambridge: Cambridge University Press.

Camacho, M. \& Lara, T. (2011). M-learning en España, Portugal y Ameirica Latina. Salamanca: Servicio de Innovación y Producción Digital de la Universidad de Salamanca.

Caudill, J. (2007). The Growth of m-Learning and the Growth of Mobile Computing: Parallel developments. International Review of
Research in Open and Distance Learning, 8 (2).

Chamasemani, F. F.\& Affendey, L. S. (2014). Impact of mobile context-aware applications on human computer interaction. Journal of Theoretical \& Applied Information Technology, 62 (1), 281-287.

Chang, C. Y., Sheu, J. P.\& Chan, T. W. (2003). Concept and Design of Ad Hoc and Mobile classrooms. Journal of Computer Assisted Learning, 19 (3), 336-346. doi: 10.1046 / j.0266-4909.00035.x

Chen, H. R.\& Huang, H. L. (2010). User Acceptance of Mobile Knowledge Management Learning System: Design and Analysis. Educational Technology \& Society, 13 (3), 70-77.

Chorfi, H. O., Sevkli, A. Z.\& Bousbahi, F. (2012). Mobile learning adaption through a device based reasoning. Procedia - Social and Behavioral Sciences, 47, 1707-1712.

Clough, G., Jones, A.C., McAndrew, P.\& Scanlon, E. (2008). Informal learning with PDAs and smartphones. Journal of Computer Assisted Learning, 24 (5), 359-371. doi: 10.1111/j.1365-2729.2007.00268.x

Crescente, M. L.\& Lee, D. (2011). Critical issues of m-learning: design models, adoption processes, and future trends. Journal of the Chinese Institute of Industrial Engineers, 28 (2), 111-123. doi: 10.1080/ 10170669.2010.548856

Dyson, L. E., Litchfield, A., Lawrence, E., Raban, R.\& Leijdekkers, P. (2009). Advancing the M-Learning Research Agenda for Active, Experiential Learning: Four Case Studies. Australasian Journal of Educational Technology, 25 (2), 250-267.

Fombona, J., Pascual, M. A.\& Madeira, M. F. (2012). Realidad aumentada, una evolución de las aplicaciones de los dispositivos móviles. Pixel-Bit, 41, 197-210. 
Georgiev, T., Georgieva, E. \& Smrikarov, A. (2004). M-Learning. A new stage of $e$ learning. Paper presented at the International Conference on Computer Systems and Technologies. Nueva York, EE.UU. doi: 10.1145/ 1050330.1050437

Hashim, K., Tan, F. B. \& Rashid, A. (2014). Adult learners' intention to adopt mobile learning: A motivational perspective. British Journal of Educational Technology. Publicacioìn anticipada en liìnea http:// onlinelibrary.wiley.com/doi/10.1111/ bjet.12148/abstract

ITU. (2011). Key ICT indicators for developed and developing countries and the world (totals and penetration rates). International Telecommunication Union.

Kakihara, M. \& Sørensen, C. (2002). Mobility: An Extended Perspective. Paper presented at the Hawai'i International Conference on System Sciences. doi: 10.1109 /HICSS.2002.994088

Koszalka, T. A. \& Ntloedibe Kuswani, G. S. (2010). Literature on the safe and disruptive learning potential of mobile Technologies. Distance Education, 31 (2), 139-157. doi: 10.1080/01587919.2010.498082

Kukulska Hulme, A. (2010). Mobile learning as a catalyst for change. Open Learning: The Journal of Open, Distance and e-Learning, 25 (3), 181-185. doi: 10.1080 / 02680513 .2010 .511945

Kukulska, A., Sharples, M., Milrad, M., Arnedillo, I. \& Vavoula, G. (2011). The Genesis and Development of Mobile Learning in Europe. In D. Parsons (Ed.).Combining ELearning and M-Learning: New Applications of Blended Educational Resources. (151-177). Hershey: Information Science Reference.
Liaw, S. S., Hatala, M. \& Huang, H. M. (2010). Investigating acceptance toward mobile learning to assist individual knowledge management: Based on activity theory approach. Computers \& Education, 54 (2), 446-454. doi: 10.1016 / j.compedu.2009.08.029

Livingston, A. (2004). Smartphones and other mobile devices: The Swiss army knives of the 21st century. EDUCAUSE Quarterly, 2, 48-52.

Sampson, D.G. \& Zervas, P. (2013). ContextAware Adaptive and Personalized Mobile Learning. Paper presented at the 3rd International Conference For e-learning \& Distance Education.

Sharples, M.\& Beale, R. (2003). A technical review of mobile computational devices. Journal of Computer Assisted Learning, 19 (3), 392-395. doi: 10.1046 / j.02664909.2003.00040.x

Sharples, M., Arnedillo, I. S., Milrad, M. \& Vavoula, G. (2007). Mobile Learning: Small devices, big issues. In N. Balacheff, S. Ludvigsen, T. de Jong, A. Lazonder \& S. Barnes (Eds.).Technology Enhanced Learning: Principles and Products. (pp.233249). Heidelberg: Springer.

Traxler, J. (2009). Current state of mobile learning. In M. Ally (Ed.).Mobile Learning: Transforming the Delivery of Education and Training. (pp. 9-24). Canada: Athabasca University.

Tsinakos, A. \& Ally, M. (2014). Global Mobile Learning Implementations and Trends. Beijing: Open University of China.

Wagner, E. (2005). Enabling mobile learning. EDUCAUSE Review, 40 (3), 40-53. 
Winters, N. (2006). What is mobile learning? In M. Sharples (Ed.).Big issues in Mobile learning: Report of a workshop by the Kaleidoscope Network of Excellence Mobile Learning Initiative (5-9). Nottingham: University of Nottingham.

Zervas, P., Gómez, S., Fabregat, R. \& Sampson, D. (2011). Tools for Context-Aware Learning Design and Mobile Delivery. Paper presented at the 11th IEEE International Conference on Advanced Learning Technologies.

Fecha de recepción: 26-08-2014

Fecha de evaluación: 28-09-2014

Fecha de aceptación: 20-10-2014 